\title{
REGISTRO DE PACIENTES CON ACCIDENTE CEREBRO VASCULAR EN UN HOSPITAL PÚBLICO DEL PERÚ, 2000-2009
}

\author{
Ana Castañeda-Guarderas ${ }^{1, a}$, Guillermo Beltrán-Ale, ${ }^{1, a}$, Renzo Casma-Bustamante, \\ Paulo Ruiz-Grosso 1,2,a, Germán Málaga ${ }^{1,3,4, b}$
}

\begin{abstract}
RESUMEN
Estudio que describe las características de los registros de pacientes con accidente cerebro vascular (ACV), como diagnóstico causante de hospitalización en el Hospital Nacional Cayetano Heredia entre los años 2000 - 2009. Se obtuvieron 2225 registros de pacientes mayores de 18 años, con ACV. De acuerdo con la CIE-10, 1071 tenían el diagnóstico de ACV isquémico, 554 ACV hemorrágico, 183 hemorragia subaracnoidea, 49 isquémico hemorrágico, 10 crisis isquémica transitorias y en 358 no fue posible especificar el tipo. Se registraron 352 muertes (19,6\%), la mayoría en los primeros tres días. La razón hombre/mujer fue 1,09; la edad promedio $64,1 \pm 17,2$ años y la mediana del tiempo de hospitalización fue de nueve días. Las condiciones asociadas más frecuentes fueron hipertensión arterial, fibrilación auricular y diabetes mellitus tipo 2. La mortalidad descrita es la más alta informada en nuestro medio, es constante en todos los grupos de edad y mayor en mujeres.
\end{abstract}

Palabras clave: Accidente cerebrovascular; Epidemiología; Hospitalización (fuente: DeCS BIREME).

\section{REGISTRY OF PATIENTS WITH STROKE STATED IN A PUBLIC HOSPITAL OF PERU, 2000-2009}

\begin{abstract}
We performed a descriptive study taking on account the characteristics of the registries of the patients hospitalized at the Hospital Nacional Cayetano Heredia between the years 2000 and 2009 with stroke as hospitalization diagnosis. 2225 records were obtained from patients older than 18, with stroke. According to ICD-10, 1071 had diagnosis of ischemic stroke, 554 were hemorrhagic, 183 were subarachnoid hemorrhage, 49 were ischemic plus hemorrhagic, 10 were transient ischemic attack (TIA) and in 358 we were unable to specify the type of stroke. 352 deaths were recorded (19.6\%), most of them during the first 3 days. The male / female ratio was 1.09 , the mean age $64.1 \pm 17.2$ years and the median length of hospital stay was 9 days. The most common associated conditions were high blood pressure, atrial fibrillation and type 2 diabetes mellitus. The mortality found is the highest reported in our country, constant in all age groups and higher in women.
\end{abstract}

Key words: Stroke; Epidemiology; Hospitalization (source: MeSH NLM).

\section{INTRODUCCIÓN}

El accidente cerebro vascular (ACV) es una causa común de muerte y discapacidad, lo cual ocasiona enormes gastos que son asumidos, en la mayor parte, por países de ingresos medios y bajos, puesto que en ellos se identifican dos terceras partes de estos casos ${ }^{(1,2)}$.

El Ministerio de Salud del Perú (MINSA) notificó un aumento de la mortalidad por ACV entre los años 2000 y $2006{ }^{\left.{ }^{3}\right)}$. Sin embargo, en el país se han realizado pocos estudios, los cuales cuentan con pocos pacientes, y difieren entre ellos y de los grandes estudios epidemiológicos internacionales. El trabajo más grande fue realizado en un hospital del seguro social el año $2000(4-6)$

El objetivo del presente trabajo fue describir las características relacionadas con el diagnóstico de ACV, la mortalidad y las particularidades de las variables asociadas, en los registros de pacientes internados en un hospital público de Perú en un período de 10 años.

\footnotetext{
Conocimiento y Evidencia (CONEVID), Universidad Peruana Cayetano Heredia. Lima, Perú.

Grupo de Trabajo en Salud Mental, Universidad Peruana Cayetano Heredia. Lima, Perú.

Centro de Excelencia en Enfermedades Crónicas (CRONICAS), Universidad Peruana Cayetano Heredia. Lima, Perú.

Departamento de Medicina, Universidad Peruana Cayetano Heredia. Lima, Perú.

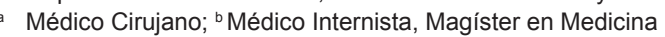

Recibido: 09-06-11 Aprobado: 12-10-11 


\section{EL ESTUDIO}

Se estudió los registros de pacientes internados en el Hospital Nacional Cayetano Heredia $(\mathrm{HNCH})$ a quienes, al dárseles el alta, se les registró con el diagnóstico de ACV, entre los años 2000 - 2009.

La información fue obtenida del Departamento de Estadística e Informática del $\mathrm{HNCH}$, correspondiente a los registros de pacientes mayores de 18 años con diagnóstico de ACV como causante de la hospitalización y clasificados en subtipos, de acuerdo con la Clasificación Internacional de Enfermedades CIE 9 o CIE 10. Los códigos corresponden a los diagnósticos definitivos asignados por el médico tratante al momento de salir de alta el paciente, opinión basada en el estudio de imágenes y otras pruebas realizadas. La información obtenida corresponde a los egresados desde el 1 de enero de 2000 al 31 de diciembre de $2009^{(7)}$.

Se excluyeron los registros de pacientes con diagnóstico de traumatismos que pudiesen haber causado el ACV, trombosis del seno venoso cerebral, secuela de ACV y en los que no figurase otro diagnóstico compatible con un nuevo episodio de ACV.

Estos registros fueron tabulados en una ficha de recolección de datos donde, además del diagnóstico, se incluyeron las condiciones asociadas como la edad, el sexo, la fecha de egreso, el tiempo de hospitalización y la condición de egreso (fallecido o no). No fue posible recolectar las características clínicas y funcionales de la población debido a que el hospital no contaba con un archivo físico que permitiera esta búsqueda de manera confiable y completa. Esta información fue registrada en una hoja de cálculo en el programa Excel 2007 @ y luego transferida al programa estadístico STATA 10.0 ® para su análisis.

Los pacientes fueron divididos en seis grupos: ACV isquémico (ACV-i) registro en el que se especifica que el tipo de $A C V$ era isquémico y no se consigna que hubiese tenido un componente hemorrágico; ACV hemorrágico
(ACV-h) registro en el que se especifica que el tipo de ACV era hemorrágico y no se consigna que hubiese tenido un componente isquémico; ACV isquémicohemorrágico (ACV-ih) registro en el que se consigna tanto el diagnóstico de ACV-i como el de ACV-h; hemorragia subaracnoidea (HSA); crisis isquémica transitoria (CIT), y ACV no especificado (ACV-ne) el cual es un registro con tipo de ACV no compatible con los descritos y en el cual no se pudo precisar el diagnóstico.

De las variables cualitativas como sexo, edad, condición de egreso, tiempo de hospitalización y condiciones asociadas, se realizó un análisis univariado con distribución de frecuencias; mientras que en las variables cuantitativas fue con medidas de tendencia central y de dispersión. Particularmente, con la variable edad los pacientes fueron agrupados por conveniencia en grupos de edad (menores de 40 años, 40 a 59 años, 60 a 79 años y de 80 años a más).

\section{HALLAZGOS}

Se hallaron 2314 registros de pacientes que cumplieron con los criterios de inclusión en el período establecido. De estos, se excluyeron a 54 pacientes por ser menores de 18 años, 16 diagnósticos por ser de etiología traumática, cinco por ser trombosis de senos venosos, once por ser diagnósticos de secuela de ACV y no un nuevo evento, y tres por haber sido incorrectamente registrados. Finalmente, 2225 registros de pacientes ingresaron a nuestro estudio.

La distribución de los registros según el tipo de $\mathrm{ACV}$, razón hombre/mujer, edad promedio, tiempo de hospitalización y mortalidad, pueden ser apreciadas en la Tabla 1. La forma de ACV más frecuente fue la de ACV isquémico, que se presento en el $48,1 \%$ de los casos, seguido por la forma de ACV-h con el 24,9\%. Proporcionalmente, la forma de ACV que presenta mayor mortalidad fue la hemorrágica con $31,8 \%$ de los casos.

También se observa que en el ACV-h se encontró una mayor proporción de varones/mujeres $(1,19)$.

Tabla 1. Características generales de pacientes con diagnóstico de ACV en un hospital público de Perú, entre 2000 a 2009.

\begin{tabular}{|c|c|c|c|c|c|}
\hline & $\mathrm{N}(\%)$ & Razón hombre/mujer & $\begin{array}{l}\text { Edad promedio } \pm \mathrm{DE} \\
\text { (años) }\end{array}$ & Tiempo hospitalización* & $\begin{array}{c}\text { Mortalidad } \\
\text { n ( \%) }\end{array}$ \\
\hline General & $2225(100)$ & 1,09 & $64,1 \pm 16,9$ & 7 & $434(19.5)$ \\
\hline ACV-i & $1071(48,1)$ & 1,08 & $65,5 \pm 16,5$ & $10(1-229)$ & $146(13,6)$ \\
\hline ACV-h & $554(24,9)$ & 1,19 & $63,1 \pm 16,4$ & $9(1-245)$ & $176(31,8)$ \\
\hline ACV-ne & $358(16,1)$ & 1,08 & $65,7 \pm 14,7$ & $8(1-142)$ & $74(20,7)$ \\
\hline HSA & $183(8,2)$ & 0,93 & $54,3 \pm 20,7$ & $8(1-203)$ & $32(17,5)$ \\
\hline $\mathrm{CIT}$ & $10(0,5)$ & 2,33 & $62,7 \pm 25,4$ & $6,5(1-16)$ & 1 (10) \\
\hline
\end{tabular}

* Mediana (Mínimo-Máximo en días)

ACV: accidente cerebro vascular, (i): isquémico, (h): hemorrágico, (ne): no especificado, (ih): isquémico hemorrágico. HSA: hemorragia subaracnoidea. CIT: crisis isquémica transitoria. 


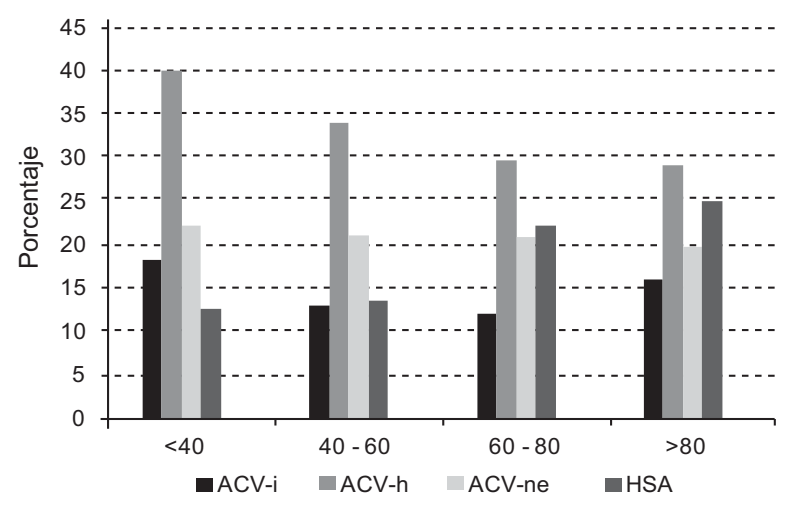

Figura 1. Porcentaje de Tipos de ACV según grupo de edad. ACV: accidente cerebro vascular, (i): isquémico, (h): hemorrágico, (ne): no especificado. HSA: hemorragia subaracnoidea

El promedio de hospitalización fue de 7 días; en la variante $A C V$-ih se encontró un mayor tiempo de hospitalización, con un promedio de 44,5 días (rango; 1-54). El mayor número de muertes ocurrió dentro de los primeros 3 días. El grupo de edad donde se halló mayor mortalidad fue el de menores de 40 años, a expensas de la forma de ACV-h, como se ve en la Figura 1; se halló mayor mortalidad en mujeres en todas las formas de ACV (Figura 2).

La condición asociada como factor de riesgo más importante para desarrollar ACV fue hipertensión arterial ( $47,7 \%$ de todos los casos), siendo discretamente más frecuente en la forma de ACV-i. En segundo lugar se encuentra la fibrilación auricular con $12,9 \%$ de los casos, siendo más frecuente en el grupo de ACV-i en el $16,9 \%$. En tercer lugar diabetes mellitus con 10,9\% de los casos y más frecuente en el grupo de ACV-i con el $14,7 \%$ de los casos como se ve en la Tabla 2 .

La condición infecciosa asociada más común en los pacientes con ACV fue la neumonía, que representa

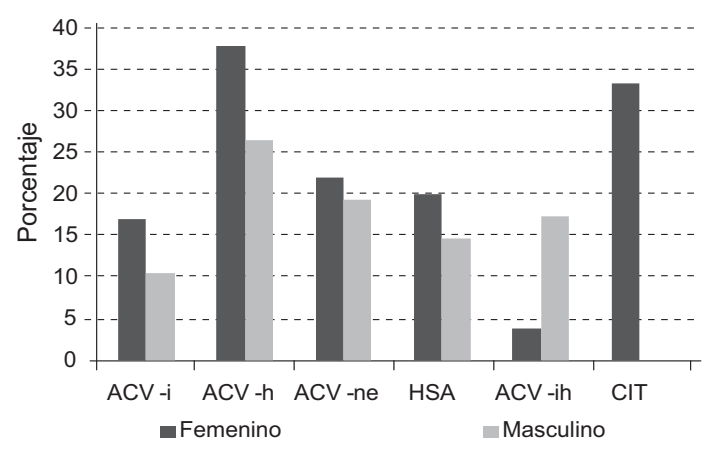

Figura 2. Porcentaje de mortalidad de acuerdo a sexo y tipo de ACV. ACV: accidente cerebro vascular, (i): isquémico, (h): hemorrágico, (ne): no especificado, (ih): isquémico hemorrágico. HSA: hemorragia subaracnoidea. CIT: crisis isquémica transitoria.

globalizada en sus variantes el $15,6 \%$, la que ocurrió con mayor frecuencia en pacientes con ACV-h $(21,2 \%)$.

Con relación a las complicaciones no infecciosas, la más frecuente fue la hipertensión endocraneana con $5 \%$, seguida de ICC en el 4,4\%, en tercer lugar insuficiencia respiratoria en el $3,9 \%$.

\section{DISCUSIÓN}

Los resultados muestran que en 10 años se tuvo 2225 casos de ACV. La mortalidad hospitalaria general fue de 19,6 \%. De los últimos cinco estudios nacionales solo dos describen la mortalidad y, en el más grande de estos, Deza et al. ${ }^{(8)}$ informan una mortalidad de $9,8 \%$ de los pacientes hospitalizados en el servicio de neurología de un Hospital del Seguro Social del Perú durante 10 años. La diferencia quizá se deba a que en el referido estudio, no se tomaron en cuenta pacientes que estuvieron en la emergencia $y$, como vimos, que la mayor cantidad de muertes ocurre

Tabla 2. Condiciones asociadas según subtipo de ACV.

\begin{tabular}{|c|c|c|c|c|c|c|c|}
\hline & & $\begin{array}{c}\text { General } \\
(\%)\end{array}$ & $\begin{array}{c}\text { ACV-i } \\
(\%)\end{array}$ & $\begin{array}{c}\text { ACV-h } \\
(\%)\end{array}$ & $\begin{array}{c}\text { ACV-ne } \\
(\%)\end{array}$ & $\begin{array}{l}\text { HSA } \\
(\%)\end{array}$ & $\begin{array}{c}\text { ACV-hi } \\
(\%)\end{array}$ \\
\hline \multirow{4}{*}{$\begin{array}{l}\text { Condiciones asociadas } \\
\text { compatibles con } \\
\text { factores de riesgo }\end{array}$} & HTA & 47,7 & 53,5 & 47,1 & 48,9 & 15,3 & 42,9 \\
\hline & Diabetes mellitus tipo 2 & 10,9 & 14,7 & 7,1 & 10,9 & 3,3 & 2,1 \\
\hline & Fibrilación auricular & 12,9 & 16,9 & 8,8 & 12,3 & 3,8 & 8,2 \\
\hline & Dislipidemia & 1,8 & 2,4 & 1,6 & 1,7 & 0 & 0 \\
\hline \multirow{4}{*}{$\begin{array}{l}\text { Condiciones asociadas } \\
\text { más comunes } \\
\text { compatibles con } \\
\text { complicaciones } \\
\text { infecciosas }\end{array}$} & Neumonía no especificada & 3,1 & 2,9 & 2,4 & 5,3 & 2,2 & 2,1 \\
\hline & Neumonía adquirida comunidad & 1,8 & 1,8 & 3,1 & 0,6 & 0,6 & 4,1 \\
\hline & Neumonía Intrahospitalaria o aspirativa & 10,7 & 10 & 15,7 & 7,3 & 3,3 & 18,4 \\
\hline & ITU & 5,9 & 7,2 & 3,9 & 8,4 & 0,6 & 6,1 \\
\hline \multirow{6}{*}{$\begin{array}{l}\text { Condiciones asociadas } \\
\text { no especificas }\end{array}$} & Falla renal no especificada & 0,2 & 0,2 & 0,4 & 0,3 & 0 & 0 \\
\hline & Insuficiencia respiratoria & 3,9 & 2,9 & 6,1 & 4,8 & 2,7 & 2,1 \\
\hline & ICC & 4,4 & 5,5 & 2,7 & 5,6 & 1,6 & 0 \\
\hline & Hipertensión Endocraneana & 5,0 & 3,3 & 7,8 & 3,6 & 10,9 & 2,0 \\
\hline & Falla renal aguda & 0,9 & 0,8 & 2,2 & 0,3 & 0,6 & 6,1 \\
\hline & Falla renal crónica & 2,9 & 3,3 & 1,9 & 3,6 & 2,2 & 0 \\
\hline
\end{tabular}

ACV: accidente cerebro vascular, (i): isquémico, (h): hemorrágico, (ne): no especificado, (ih): isquémico hemorrágico. HSA: hemorragia subaracnoidea. 
en los primeros días, estas muertes pudieran no haber sido registradas. Con relación a otros estudios internacionales (EE.UU. (9) y Argentina (10) $)$ nuestra mortalidad es mayor, pero, puede ser que la comparación no sea definitiva, pues en el estudio estadounidense se incluyó a pacientes con diagnóstico de CIT (casi el $25 \%$ de la muestra) y en el estudio argentino solo evaluaron pacientes con ACV-i que, en general, tiene una menor mortalidad. La falta de una unidad especializada en ACV en el hospital o la falta de implementación de protocolos de administración de terapia trombolítica, pudieran explicar perfectamente estos resultados. Sin embargo, si tomamos en cuenta el informe de la $\mathrm{AHA}^{(11)}$ el cual menciona una mortalidad de 18,4\%, o el $22,9 \%$ de mortalidad a 30 días informado en un estudio epidemiológico de América Latina y el Caribe ${ }^{(12)}$, los resultados presentados no son muy distintos.

Llama la atención el discreto aumento de los casos de ACV a lo largo de los 10 años estudiados, hecho que sorprende si se tiene en consideración las sospechas de incremento de enfermedades crónicas en nuestro medio.

Un hallazgo inesperado fue la mayor mortalidad en el grupo de menores de 40 años dentro del grupo de ACV-h (Figura 1), sin embargo, así no fuera significativa la mortalidad general y por tipos de ACV se mantiene constante dentro de todos los grupos de edad. Este patrón es diferente al que describen Fonarow et al. (9) en EE.UU., en donde conforme la edad aumenta, se incrementa el riesgo de muerte intrahospitalaria independientemente del tipo de ACV. Este comportamiento no había sido descrito previamente en nuestro medio ni en Latinoamérica y no se puede plantear una única explicación; sin embargo, antes de asimilarlo, se debería impulsar el diseño de un estudio que proporcione información necesaria y razonable que ratifique o deseche este hallazgo, pues se podrían postular una serie de de factores como una mayor gravedad del ACV en pacientes jóvenes, mayor número de registros de ACV como causa de muerte en pacientes jóvenes, el tiempo que demora el paciente en llegar al hospital y la capacidad resolutiva del hospital, entre otros.

Así mismo, se halló que la mortalidad fue mayor en las mujeres y en ACV-h (Figura 2), no obstante a nivel internacional, esta asociación: "sexo y mortalidad" no ha sido descrita ${ }^{(13-17)}$.

La razón hombre/mujer cercana a $1(1,09)$ es similar a lo descrito en estudios realizados en EE.UU. $(0,89)$, Reino Unido $(1,2-1,4)$ y Argentina $(1,35)^{(9,10,18)}$. En base a este resultado nuestra población se comportaría de acuerdo con lo descrito por la $A H A{ }^{(11)}$, la cual afirma que la incidencia de ACV es mayor en varones; sin embargo, difiere de estudios nacionales, los que a su vez son discordantes, pues Deza et al. ${ }^{\left({ }^{8}\right)}$ informaron una marcada diferencia de 2,21 , mientras que Astorga et al. (19) mostraron una razón de 0,45 ; esto probablemente se deba a una mayor atención al hombre o a la mujer en los hospitales donde se realizaron los estudios, o a que se trató de muestras no probabilísticas.

En los estudios contemplados en la revisión sistemática de Latinoamérica ${ }^{(20)}$ se informó una proporción de ACV-h mayor que la descrita en EE.UU. ${ }^{(9)}$ y Europa ${ }^{(18)}$. Incluso, dentro del estudio americano, hay mayor proporción de ACV-h en hispanos que en la población general, al igual que cuando se comparó argentinos blancos con nativos en Buenos Aires. Para los fines del análisis se excluyó a los pacientes con HSA como se suele realizar en otros estudios, resultando una proporción de ACV-h de 33,1 \%, dato similar a los estudios latinoamericanos pero mayor que los países desarrollados y los estudios nacionales previos. Esta diferencia podría estar relacionada con factores genéticos, a diferentes factores de riesgo y otros no totalmente esclarecidos.

En lo que respecta al tiempo de hospitalización, se observa que el $50 \%$ de los pacientes estudiados permanece, por lo menos, nueve días; dentro de ellos, la mitad de los pacientes con ACV-i se queda por lo menos diez días. Este tiempo de hospitalización es mayor que el descrito en estudios norteamericanos (9) y sudamericanos, como el ReNACer de Argentina (10). Estudios previamente realizados en nuestro país no han estudiado esta variable ${ }^{(8,19,21)}$. Probablemente el mayor tiempo de hospitalización hallado está relacionado con la falta de recursos de la población atendida en el $\mathrm{HNCH}$, lo cual retrasa la realización de pruebas de apoyo diagnóstico y seguimiento necesarias para esclarecer la etiología del evento, lo que prolonga su estadía.

La principal fortaleza del presente estudio es que se ha recogido el mayor número de atenciones de pacientes con ACV en un hospital del MINSA, de donde se puede obtener características generales de la población así como información epidemiológica relevante para futuras investigaciones. Sin embargo, dado que nuestra fuente de información es un registro de egresos, el estudio cuenta con limitaciones como la falta de información del método diagnóstico, que un mismo paciente se haya registrado más de una vez y que se presenta un alto porcentaje alto de registros $(16,1 \%)$ sin especificación del subtipo de $A C V$, situación que también fue descrita en el estudio epidemiológico de Latinoamérica y el Caribe ${ }^{(12)}$ los que podrían tener, incluso, diagnósticos alternos al ACV. 
Basados en los resultados obtenidos se recomienda mejorar el sistema de registro del $\mathrm{HNCH}$, así como realizar estudios prospectivos que permitan esclarecer por qué la estancia hospitalaria es tan prolongada; verificar si en efecto la mortalidad es tan alta como la reportada, si esta no varía entre grupos de edad y si es mayor en el sexo femenino; obtener información sobre la calidad de vida de pacientes afectados con ACV, su diversa morbilidad y mortalidad y, sobre todo, establecer las medidas e intervenciones que permitan reducir la mortalidad hospitalaria, mejorar la funcionalidad, su calidad de vida, prevenir nuevos eventos y, en general, mejorar los resultados de la evolución de estos pacientes.

\section{Contribuciones de autoría}

Todos los autores participaron de la concepción y diseño, recolección, análisis, revisión crítica del manuscrito, y de la aprobación de versión final. PRG realizó la asesoría estadística. GM brindó el material de estudio.

\section{Conflictos de interés}

Los autores declaran no tener conflictos de interés.

\section{Fuentes de financiamiento}

Autofinanciado.

\section{REFERENCIAS BIBLIOGRÁFICAS}

1. Bonita R, Mendis S, Truelsen T, Bogousslavsky J, Toole J, Yatsu F. The global stroke initiative. Lancet Neurol. 2004;3:391-3

2. Menken M, Munsat TL, Toole JF. The global burden of disease study: implications for neurology. Arch Neurol. 2000;57:418-20.

3. Oficina General de Estadística e Informática. Ministerio de Salud. Principales causas de mortalidad por sexo. Lima: INEI; 2006.

4. Jaillard AS, Hommel M, Mazetti P. Prevalence of stroke at high altitude $(3380 \mathrm{~m})$ in Cuzco, a town of Peru. A population-based study. Stroke 1995;26:562-8.

5. Neira Sanchez ER, Seclen Santiesteban S. Estudio retrospectivo de la ocurrencia de accidente cerebrovascular en pacientes diabéticos. Rev Med Hered. 2000;11(2):54-61.

6. Córdova Ruiz ME. Factores de riesgo modificables para un primer evento de enfermedad vascular cerebral isquémica, en el Hospital Alberto Sabogal Sologuren, en el periodo enero 2002 - diciembre 2002. Tesis para optar el título de médico especialista en neurología. Lima Perú: Facultad de Medicina, Universidad Nacional Mayor de San Marcos. 2005.

7. Hospital Nacional Cayetano Heredia. Oficina de Epidemiología y Salud Ambiental. Lima: $\mathrm{HNCH}$; $c 2011$.

8. Deza L AR, Barrera J. Historia Natural de la Enfermedad Vascular Cerebral en el Perú - Estudio intrahospitalario de 1517 pacientes. Rev NeuroPsiquiat. 2001;64(2):105-32.
9. Fonarow GC, Reeves MJ, Smith EE, Saver JL, Zhao X, Olson DW, et al. Characteristics, performance measures, and in-hospital outcomes of the first one million stroke and transient ischemic attack admissions in get with the guidelines-stroke. Circ Cardiovasc Qual Outcomes. 2010;3(3):291-302.

10. Sposato LA, Esnaola MM, Zamora R, Zurru MC, Fustinoni O, Saposnik G. Quality of ischemic stroke care in emerging countries: the Argentinian National Stroke Registry (ReNACer). Stroke. 2008;39:3036-41.

11. Goldstein LB, Bushnell CD, Adams RJ, et al. Guidelines for the primary prevention of stroke: a guideline for healthcare professionals from the American Heart Association/ American Stroke Association. Stroke. 2011;42(2):517-84.

12. Lavados $\mathbf{P}$, Fernandez J, Medina M, Legetic B. Stroke epidemiology, prevention, and management strategies ar a regional level: Latin America and the Caribbean. Lancet Neurol. 2007;6(4):362-72.

13. Vaartjes I, Reitsma JB, Berger-van Sijl M, Bots ML. Gender differences in mortality after hospital admission for stroke. Cerebrovasc Dis. 2009;28(6):564-71.

14. Ayala C, Croft JB, Greenlund KJ, Keenan NL, Donehoo RS, Malarcher AM, et al. Sex differences in US mortality rates for stroke and stroke subtypes by race/ethnicity and age, 1995-1998. Stroke. 2002;33(5):1197-201.

15. Di Carlo A, Lamassa M, Baldereschi M, Pracucci G, Basile AM, Wolfe CD, et al. Sex differences in the clinical presentation, resource use, and 3-month outcome of acute stroke in Europe: data from a multicenter multinational hospital-based registry. Stroke. 2003;34(5):1114-9.

16. Sheikh K, Bullock CM. Effect of measurement on sex difference in stroke mortality. Stroke. 2007;38(3):1085-7.

17. Holroyd-Leduc JM, Kapral MK, Austin PC, Tu JV. Sex differences and similarities in the management and outcome of stroke patients. Stroke. 2000;31(3):1833-7.

18. Bhatnagar $P$, Scarborough $P$, Smeeton NC, Allender S. The incidence of all stroke and stroke subtype in the United Kingdom, 1985 to 2008: a systematic review. BMC Public Health. 2010;10:539.

19. Astorga Castillo JL. Aspectos clinico epidemiológicos de la enfermedad cerebrovascular. Servicio de emergencia del Hospital Arzobispo Loayza. Tesis para optar el título de médico especialista en medicina de emergencias y desastres. Facultad de Medicina, Universidad Nacional Mayor de San Marcos. 2005.

20. Saposnik G, Del Brutto OH; Iberoamerican Society of Cerebrovascular Diseases. Stroke in South America: a systematic review of incidence, prevalence, and stroke subtypes. Stroke. 2003;34(9):2103-7.

21. O’Donnell M, Liu L, Zhang H, Chin SL, Rao-Melacini $\mathbf{P}$, Rangarajan $\mathbf{S}$, et al. Risk factors for ischaemic and intracerebral haemorrhagic stroke in 22 countries (the INTERSTROKE study): a case-control study. Lancet. 2010;376(9735):112-23.

Correspondencia: Germán Málaga Rodríguez

Dirección: Calle Chavín 159 CC Monterrico. Surco. Lima 33.

Perú.

Teléfono: (511)-3445356

Correo electrónico: german.malaga@upch.pe 